\title{
A simple method to recover the graphene-based chemi-resistor signal
}

\author{
F. Fedi ${ }^{1,4}$, F. Ricciardella ${ }^{2,3}$, M. L. Miglietta ${ }^{2}$, T. Polichetti ${ }^{2}$, E. Massera ${ }^{2}$, and G. Di Francia ${ }^{2}$ \\ ${ }^{1}$ CNR-Institute for Composite and Biomedical Materials, Portici (Naples), Italy \\ ${ }^{2}$ ENEA UTTP-MDB Laboratory, R. C. Portici (Naples), Italy \\ ${ }^{3}$ University of Naples "Federico II", Department of Physics, Naples, Italy \\ ${ }^{4}$ Faculty of Physics, University of Vienna, Strudlhofgasse 4, 1090 Vienna, Austria
}

Correspondence to: F. Ricciardella (filiberto.ricciardella@enea.it)

Received: 21 July 2014 - Revised: 21 September 2014 - Accepted: 23 September 2014 - Published: 9 October 2014

\begin{abstract}
We present the development of a simple and fast method for restoring exhaust graphene-based chemiresistors used for $\mathrm{NO}_{2}$ detection. Repeatedly exposing the devices to gases or to air for more than 2 days, an overall worsening of the sensing signal is observed; we hypothesized that the poisoning effect in both cases is caused by the exposure to $\mathrm{NO}_{2}$. Starting from this hypothesis and from the observation that $\mathrm{NO}_{2}$ is soluble in water, we performed a recovery method consisting in the dipping of exhaust devices into ultrapure water at $100^{\circ} \mathrm{C}$ for $60 \mathrm{~s}$. The device performances are compared with those obtained after the restoration is achieved using the typical annealing under vacuum method.
\end{abstract}

\section{Introduction}

A crucial point for solid-state gas sensor use at room temperature (RT) is the difficulty of recovery at the initial conditions after the sensing operation (Schedin et al., 2007; Yavari and Koratkar, 2012; Yuan and Shi, 2013). The drawback arises from the interaction energy between sensitive materials and gases, as reported by conventional transition state theory. At RT, in fact, the energies involved in the adsorption phenomena are in the range of $\mathrm{eV}$, so that, once chemisorbed, reversibility is not thermodynamically favored. In order to allow the formation of a free interface on the sensitive layer, gas molecules generally have to be removed by recovery methods, supplying an external source of energy and UV irradiation, thermal treatment and electric field applications; all the above mentioned methods have been proposed for this purpose (Chen et al., 2001; Hyman and Medlin, 2005; Schedin et al., 2007; Charlier et al., 2009; Leghrib and Llobet, 2011). Chen et al. (2001) continuously apply cleaning in situ on the sensing material by pumping ultraviolet light, achieving the record gas detection of parts per trillion (ppt), although in an inert atmosphere. Even Schedin et al. (2007) found that ultraviolet irradiation or annealing at $150{ }^{\circ} \mathrm{C}$ can restore the state of the devices after analyte exposure, although under vacuum. In Charlier et al. (2009), the carbon nanotube (CNT) based sensors were kept heated at $150{ }^{\circ} \mathrm{C}$ to speed up gas desorption, while the test chamber was flushed with pure dry air for $1 \mathrm{~h}$ after each series of successive injections; when the airflow was interrupted, the sensors were left at ambient temperature for $12 \mathrm{~h}$ so that the full recovery of baseline resistance was reached. In this case, operating at RT, the $\mathrm{NO}_{2}$ minimum concentration of $500 \mathrm{ppb}$ was achieved. Leghrib and Llobet raised the temperature of the CNT-based sensors to $150^{\circ} \mathrm{C}$, promoting the cleaning after the gas exposure, while dry air was injected in the chamber. Therefore, the standard approach is essentially based on the increase of the analyte molecule mobility by providing the energy to improve the adsorbate desorption from the sensitive layer. For the specific sensor device investigated in this work, we propose a new refreshing method that relies on the capability of removing the $\mathrm{NO}_{2}$ through water, the most suitable solvent for this target analyte (Tan and Piri, 2013). The chemi-resistor performances towards 350 parts per billion ( $\mathrm{ppb}$ ) of $\mathrm{NO}_{2}$ in wet $\mathrm{N}_{2}$ environment (relative humidity $=50 \%$, temperature $=22^{\circ} \mathrm{C}$ ) were compared before and after the restoration process. The proposed method 


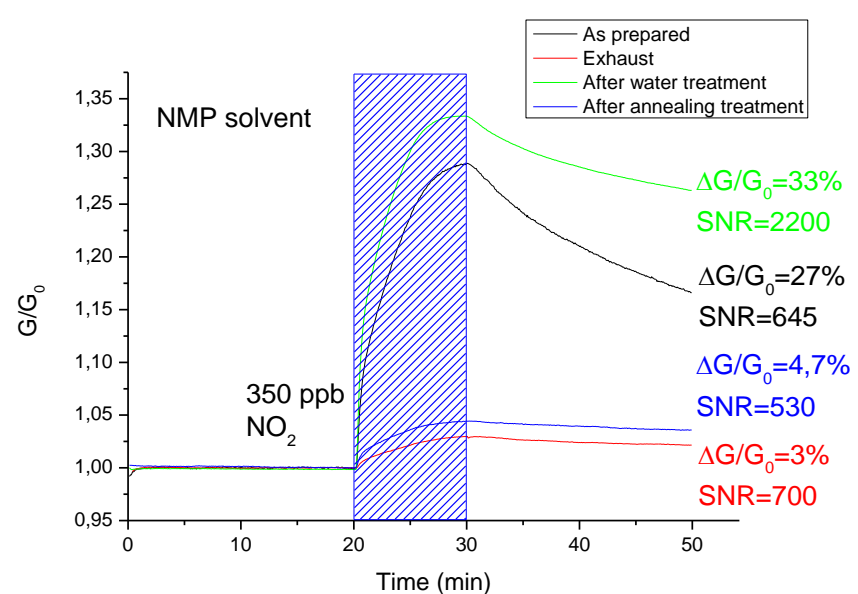

Figure 1. Normalized electrical conductance behaviors for chemiresistors towards $350 \mathrm{ppb}$ of $\mathrm{NO}_{2}$ in a wet $\mathrm{N}_{2}$ environment as soon as prepared (black line), after one month (red line), after the restoration by the dipping method (green line), and after the restoration by the annealing method (blue line). The standard protocol adopted in these tests consists of the baseline of $20 \mathrm{~min}$ in wet $\mathrm{N}_{2}$, the window exposure of $10 \mathrm{~min}$ to $\mathrm{NO}_{2}$ (blue dashed color area in the figures), and the recovery phase of $10 \mathrm{~min}$. The graphene was exfoliated using NMP as a solvent.

was tested on devices prepared starting from two different graphene solutions, and the results were also compared with those obtained by using the thermal treatment at $130^{\circ} \mathrm{C}$ in vacuum.

\section{Materials and methods}

Colloidal graphene suspensions were prepared by the liquid phase exfoliation (LPE) method. Graphite flakes (SigmaAldrich, product no. 332461) at $2.5 \mathrm{~g} \mathrm{~L}^{-1}$ were dispersed in NMP (N-methyl-pyrrolidone, Sigma-Aldrich, product no. 328634) or in a mixture of isopropanol and n-butanol (IPA/n$\mathrm{BuOH})$. Then, a mild sonication treatment was required for $168 \mathrm{~h}$ at a low power aiming to promote the graphite exfoliation (Fedi et al., 2014; Khan et al., 2010). Films prepared from the colloidal suspension were characterized as reported in Khan et al. (2010), confirming the presence of a few layers of graphene (FLG).

In order to fabricate the chemi-resistor devices, a few microliters of the colloidal dispersion were deposited by dropcasting directly onto alumina substrates with interdigitated Au electrodes (Fedi et al., 2014).

\section{Chemi-resistor testing}

The chemi-resistors were tested in a Gas Sensor Characterization System (GSCS, Kenosistec equipment) under $\mathrm{N}_{2}$ gas flow at atmospheric pressure, temperature and relative humidity (RH) set at $22^{\circ} \mathrm{C}$ and $50 \%$, respectively. The standard exposure protocol adopted for each measurement consists

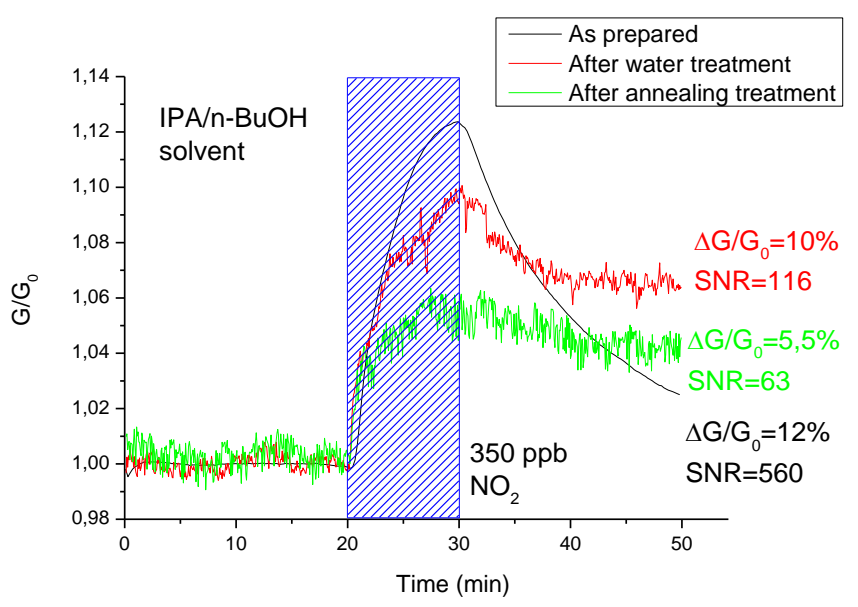

Figure 2. Normalized electrical conductance behaviors for chemiresistors towards $350 \mathrm{ppb}$ of $\mathrm{NO}_{2}$ in a wet $\mathrm{N}_{2}$ environment as soon as prepared (black line), after the restoration by the dipping method (red line), and after the restoration by the annealing method (green line). The standard protocol adopted in these tests consists of the baseline of $20 \mathrm{~min}$ in wet $\mathrm{N}_{2}$ and the window exposure of $10 \mathrm{~min}$ to $\mathrm{NO}_{2}$ (blue dashed color area in the figures). In this case, the graphene solution was prepared by exfoliating graphite flakes in a mixture of IPA/n-BuOH solvent.

basically of three steps: (a) the baseline, during which only the carrier gas is fluxed with the aim of stabilizing the current; (b) the exposure window to the target gas; and (c) the recovery phase, during which the analyte flow is stopped and again only the carrier gas is fluxed in order to return the device to the initial conditions (Figs. 1-2) (Massera et al., 2014; Ricciardella et al., 2014).

The devices were tested at three different steps: as soon as prepared, after about one month, during which they were exposed in air, and finally after applying the two described restoring methods, namely the device annealing at $130^{\circ} \mathrm{C}$ in vacuum for $120 \mathrm{~min}$ and the newly developed method that encompasses the dipping of exhaust devices into ultrapure water at $100^{\circ} \mathrm{C}$ for $60 \mathrm{~s}$, followed by a drying step on the hot plate at $150^{\circ} \mathrm{C}$ for $5 \mathrm{~min}$.

\section{Results and discussion}

To remove the adsorbed molecules from the sensitive sites, the proposed method takes advantage of the strong solubility of $\mathrm{NO}_{2}$ in $\mathrm{H}_{2} \mathrm{O}$. This dissolution mechanism is well known in the literature, as explained by Tan and Piri (Tan and Piri, 2013). $\mathrm{NO}_{\mathrm{x}}$ is a mixture of all nitrogen oxides $\left(\mathrm{N}_{2} \mathrm{O}, \mathrm{NO}\right.$, $\mathrm{NO}_{2}, \mathrm{~N}_{2} \mathrm{O}_{3}, \mathrm{~N}_{2} \mathrm{O}_{4}$, and $\mathrm{N}_{2} \mathrm{O}_{5}$ ), most of which immediately react with water upon dissolution, resulting in $\mathrm{HNO}_{3}$ and $\mathrm{HNO}_{2}$ formation. Only $\mathrm{N}_{2} \mathrm{O}$ (nitrous oxide) and NO (nitric oxide) do not react and hardly dissolve in water according to the following equilibrium reaction: $3 \mathrm{NO}_{2}+\mathrm{H}_{2} \mathrm{O} \leftrightarrow$ $2 \mathrm{HNO}_{3}+\mathrm{NO}$. 

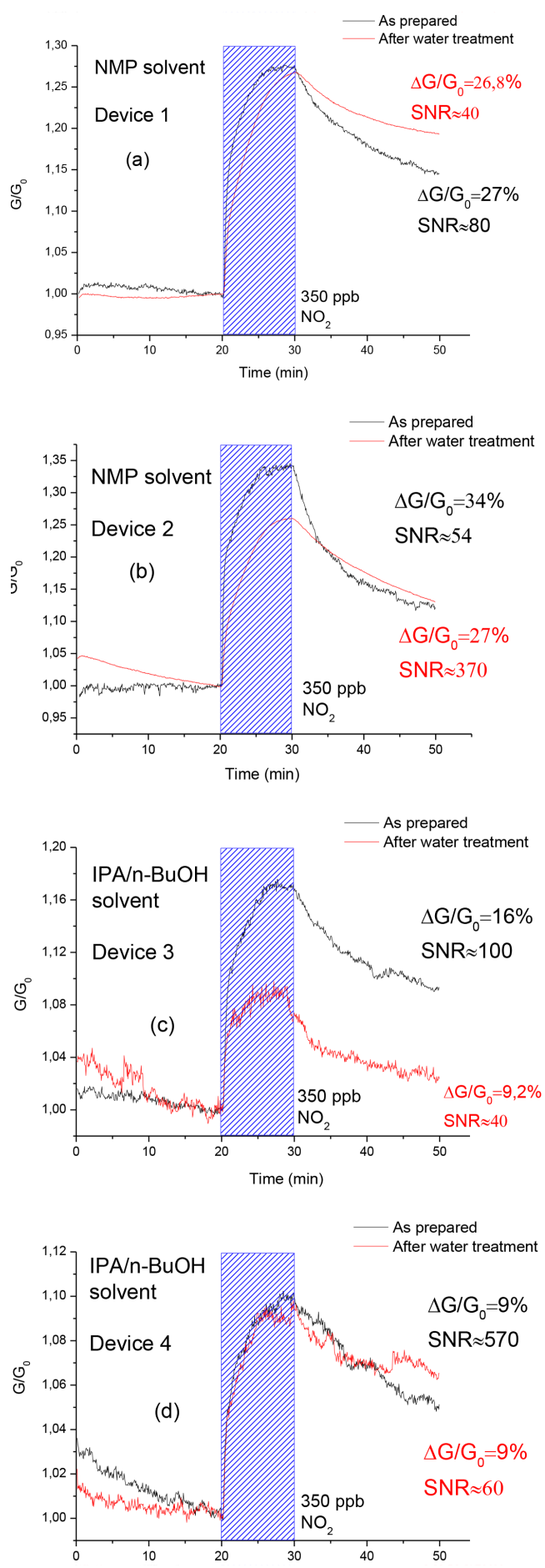

Figure 3. Normalized electrical conductance behaviors for another four chemi-resistors that have been realized as described in Fig. 1 (devices 1 and 2) and in Fig. 2 (devices 3 and 4). The black and red lines in each panel report the detected device signal as soon as prepared and after the restoration by the dipping method, respectively. The protocol employed in the tests is the standard one adopted for the previous measurements.
In Fig. 1, the normalized conductance behaviors of chemiresistors exposed to $\mathrm{NO}_{2}$ vs. the acquisition time are reported, $G_{0}$ being the initial value during the gas inlet (Fedi et al., 2014). Tests in Fig. 1 referred to devices prepared with graphene exfoliated in NMP. The stacked curves are related to tests performed at different times on the same device.

It is straightforward to observe the effects of the restoring approaches on the graphene-based devices. After the first test, which was carried out as soon as the solution was spread onto the transducers (black line), the chemi-resistor was stored in a covered petri dish and left in air. When it was tested again after one month, the variation of the device conductance towards the same analyte concentration and test conditions dramatically decreased (3\% vs. $27 \%$ ) (red line), maybe due to the strong poisoning effect that occurred in that period by the exposure to contaminants present in the atmosphere, $\mathrm{NO}_{2}$ included. The device was refreshed by using the water-based restoration approach proposed here and then exposed again using the same measurement protocol. A definite increase in $\Delta G / G_{0}$ (green line) was then observed with respect to the behavior exhibited by the exhaust device (33\% vs. $3 \%$ ). Moreover, the conductance variation resulted in being even higher than the value provided by the freshly prepared device. The response (Fig. 1c) clearly shows how the refresh method has beneficial effects on the device, suggesting water-removing effects on the $\mathrm{NO}_{2}$ molecules.

Our approach was compared with the method usually adopted in the literature (Schedin et al., 2007). The restoration in vacuum at $130^{\circ} \mathrm{C}$ for $120 \mathrm{~min}$ was carried out after leaving the device another month in air. Figure 1 (blue line) reports the behavior exposing the chemi-resistor at $350 \mathrm{ppb}$ of $\mathrm{NO}_{2}$. Since the conductance variations are similar to those measured with the exhaust device (red line in Fig. 1), it is straightforward to see the validity of the first recovery approach with respect to annealing under vacuum.

An identical procedure was accomplished on a chemiresistor based on graphene colloidal dispersion prepared by using IPA/n-BuOH solvent instead of NMP, and in Fig. 2 their normalized conductance is reported.

Figure 2 (black line) shows the conductance of the chemiresistors exposed to $\mathrm{NO}_{2}$ as soon as prepared. Differently from the previous case, when the device was left in air and then tested again, an overall absence of signal (not reported) was observed, indicating the occupancy of all sensing sites by the adsorbates. The red and green lines in Fig. 2, respectively, report on the tests after dipping and vacuum-heat restoring methods, respectively. Once more, the result (red line in Fig. 2) confirms that the developed method is able even to restore sensing layers fully insensitive to $\mathrm{NO}_{2}$. At the same time, the green line in Fig. 2 shows that the proposed method restores the sensing layer performances more efficiently than the thermal one. In some cases, the water approach can not only restore the exhaust devices, but it is even able to enhance the sensing capability towards analyte. 
The comparison between Fig. 1 (green line) and Fig. 2 (red line) clearly shows a remarkable difference related to both the conductance and the signal-to-noise ratio (SNR) values. As a matter of fact, since NMP has been demonstrated to be the most efficient solvent for exfoliating graphene (Khan et al., 2011), a larger yield of graphene flakes is actually induced by this solvent with respect to any other. As a result, SLG and FLG interconnections also increase, resulting in the enhancement of signals and an improvement in the SNR.

In Fig. 3, the tests performed on other two series of devices are reported. Figure $3 \mathrm{a}-\mathrm{b}$ and $\mathrm{c}-\mathrm{d}$ refer to devices based on graphene suspension dissolved in NMP and IPA/n-BuOH solvent, respectively. The reproducibility of the restoration process for the developed approach is confirmed in all four experiments. In some cases, the full restoration can be obtained and the initial conditions recovered, as can be observed for Device 4 (Fig. 3d).

\section{Conclusions}

In summary, an easy, original method to refresh the exhaust graphene-based chemi-resistor after $\mathrm{NO}_{2}$ exposure or after storage in air has been introduced. This novel approach basically derives from the ability of water to remove the adsorbed $\mathrm{NO}_{2}$ molecules. The method effectiveness is demonstrated by the fact that devices fully unreactive to the analyte, after the restoration, show performances not only comparable to but in some cases even better than those obtained when the device is freshly prepared.

Edited by: M. Meyyappan

Reviewed by: two anonymous referees

\section{References}

Charlier, J. C., Arnaud, L., Avilov, I. V., Delgado, M., Demoisson, F, Espinosa, E. H., Ewels, C. P., Felten, A., Guillot, J., Ionescu, R., Leghrib, R., Llobet, E., Mansour, A., Migeon, H. N., Pireaux, J. J., Reniers, F., Suarez-Martinez, I., Watson, G. E., and Zanolli, Z.: Carbon nanotubes randomly decorated with gold clusters: from nano ${ }^{2}$ hybrid atomic structures to gas sensing prototypes, Nanotechnology, 20, 375501, doi:10.1088/09574484/20/37/375501, 2009.

Chen, R. J., Franklin, N. R., Kong, J., Cao, J., Tombler, T. W., Zhang, Y., and Dai, H.: Molecular photodesorption from singlewalled carbon nanotubes, Appl. Phys. Lett., 79, 2258-2260, 2001.

Fedi, F., Ricciardella, F., Polichetti, T., Miglietta, M. L., Massera, E., and Di Francia, G.: Exfoliation of Graphite and Dispersion of Graphene in Solutions of Low-Boiling-Point Solvents for Use in Gas Sensors, in: Sensors and Microsystems, Springer, 2014.

Hyman, M. P. and Medlin, J. W.: Theoretical study of the adsorption and dissociation of oxygen on $\mathrm{Pt}(111)$ in the presence of homogeneous electric fields, J. Phys. Chem. B, 109, 6304-6310, 2005.

Khan, U., O’Neill, A., Lotya, M., De, S., and Coleman, J. N.: HighConcentration Solvent Exfoliation of Graphene, Small, 6, 864871, 2010.

Khan, U., Porwal, H., O’Neill, A., Nawaz, K., May, P., and Coleman, J. N.: Solvent-exfoliated graphene at extremely high concentration, Langmuir, 27, 9077-9082, 2011.

Leghrib, R. and Llobet, E.: Quantitative trace analysis of benzene using an array of plasma-treated metal-decorated carbon nanotubes and fuzzy adaptive resonant theory techniques, Anal. Chim. Acta, 708, 19-27, 2011.

Massera, E., Miglietta, M. L., Polichetti, T., Ricciardella, F., and Di Francia, G.: Reproducibility of the Performances of GrapheneBased Gas-Sensitive Chemiresistors, in: Sensors and Microsystems, Springer, 2014.

Ricciardella, F., Massera, E., Polichetti, T., Miglietta, M. L., and Di Francia, G.: A calibrated graphene-based chemi-sensor for sub parts-per-million $\mathrm{NO}_{2}$ detection operating at room temperature, Appl. Phys. Lett., 104, 183502, doi:10.1063/1.4875557, 2014.

Schedin, F., Geim, A., Morozov, S., Hill, E., Blake, P., Katsnelson, M., and Novoselov, K.: Detection of individual gas molecules adsorbed on graphene, Nat. Mater., 6, 652-655, 2007.

Tan, S. P. and Piri, M.: Modeling the Solubility of Nitrogen Dioxide in Water Using Perturbed-Chain Statistical Associating Fluid Theory, Ind. Eng. Chem. Res., 52, 16032-16043, 2013.

Yavari, F. and Koratkar, N.: Graphene-Based Chemical Sensors, J. Phys. Chem. Lett., 3, 1746-1753, 2012.

Yuan, W. and Shi, G.: Graphene-based gas sensors, J. Materials Chem. A, 1, 10078-10091, 2013. 